CIRJE-F-852

\title{
Congestion, Technical Returns, and the Minimum Efficient Scales of Local Public Expenditures: An Empirical Analysis for Japanese Cities
}

\author{
Masayoshi Hayashi \\ University of Tokyo
}

May 2012

CIRJE Discussion Papers can be downloaded without charge from:

$\underline{\text { http://www.cirje.e.u-tokyo.ac.jp/research/03research02dp.html }}$

Discussion Papers are a series of manuscripts in their draft form. They are not intended for circulation or distribution except as indicated by the author. For that reason Discussion Papers may not be reproduced or distributed without the written consent of the author. 


\title{
Congestion, technical returns, and the minimum efficient scales of local public expenditures: An empirical analysis for Japanese cities*
}

\author{
Masayoshi Hayashi \\ Faculty of Economics, The University of Tokyo \\ Hongo 7-3-1, Bunkyo-ku, Tokyo 113-0033, Japan \\ E-mail: hayashim at e.u-tokyo.ac.jp \\ Phone: $+81-3-5841-5513$ (DI) \\ Fax: +81-3-5841-5521
}

\begin{abstract}
On the basis of the standard model of local public production, we delineate the factors that account for the "U-shaped" per capita local public expenditures and relate them to construct an efficiency indicator for local populations. We articulate that population-induced changes in the per capita cost are related to the relative magnitude between the (i) technical elasticity of scale, which characterizes technology for the direct outputs produced by a government, and (ii) congestion elasticity, which characterizes consumption technology for the public services consumed by citizens. Those two elasticities allow us to construct an indicator that quantifies the distance of a local population from its minimum efficient scale (MES) for local public expenditures. We then estimate the urban public production structure in Japan and apply the analysis to the Japanese case. With the estimates obtained, we rank the Japanese cities according to the calculated values of the indicator.
\end{abstract}

Key words: public production, returns to scale, congestion, minimum efficient scale, local public expenditures

JEL Codes: H72, R59

\footnotetext{
* This is a reproduction of Hayashi (2002) [Hayashi, M., 2002, Congestion, technical returns, and the minimum efficient scales of local public expenditures: The case of Japanese municipalities. Discussion Paper Series 01-01, Institute for Research in Business and Economics (IRBE), Meiji Gakuin University], which has been cited by other studies several times but have been unavailable from the IRBE. While the text in this discussion paper has been edited, the theoretical analysis and the estimation results have been kept intact as those estimated in the original discussion paper above.
} 


\section{Introduction}

In standard economics terminology, "minimum efficient scale" (or "MES") refers to the scale where the average cost of production is minimized. Since Hirsh (1959) raised the issue of possible economies of scale by estimating the "U-shaped" per capita local public expenditures, the measurement of the MES of a population, as well as returns to scale in local public production, has become one of the popular topics in the literature. Despite a number of studies that have examined returns to scale in the production of local public services, however, less consideration has been given to relating the factors that affect the returns to scale to the construction of an indicator for the efficient scales of local public production. In this paper, we articulate the factors that account for the "U-shaped" per capita local public expenditures and apply the analysis to construct an indicator that quantifies a deviation from the MES of a local population for public expenditures. Our theoretical model borrows from the standard model (e.g., Bradford et al. 1969, Brueckner 1981, Duncombe and Yinger 1993), which distinguishes between the direct outputs produced by a government and the level of the public services actually consumed by citizens. In our analysis, two important concepts are the (i) technical elasticity of scale, which characterizes production technology for the direct output, and (ii) congestion elasticity, which characterizes consumption technology for the public services. By articulating that population-induced changes in per capita cost are related to the relative magnitude between the two elasticities, we derive an indicator for efficiency that quantifies the distance of a local population from its MES, an indicator that is just a simple ratio of the congestion elasticity and the technical scale elasticity. We then apply the analysis to obtain the efficient scale indicators for the Japanese cities, which are subsequently ranked according to the results.

\section{The Model}

We employ the standard model of local public production (e.g., Bradford et al. 1969, Brueckner 1981, Duncombe and Yinger 1993). This model distinguishes between the direct outputs produced by a government and the public service level consumed by citizens, which implies that the public production-consumption process can be decomposed into two stages. 
The first stage is analogous to the standard theory of the firm. As in private production, a local government produces a direct output $g$, with a technology:

$$
g=g(x)
$$

where $\boldsymbol{x}$ is a vector of factor inputs. The expenditure (or cost) function is thus derived as:

$$
c=c(g, \boldsymbol{w}) \equiv \min \left\{\boldsymbol{w}^{\prime} \boldsymbol{x} \mid g(\boldsymbol{x})=g\right\}
$$

where $w$ a vector of factor input prices. The standard properties apply to functions (1) and (2).

The second stage transforms the direct output $g$ into its service level $z$, which is of interest to citizen-consumers. Note that this transformation may be influenced by the number of consumers (i.e., population) $n$ and other local characteristics or environmental factors $\boldsymbol{a}$. This relation, known as the "crowding function" or "congestion function," is expressed as:

$$
z=z(g, n, \boldsymbol{a})
$$

where $\partial z / \partial g>0$ and $\partial z / \partial n<0$. From this, we derive the following "inverse" function with respect to $g$ :

$$
g=\gamma(z, n, \boldsymbol{a}) \equiv z^{-1}(z, n, \boldsymbol{a}) .
$$

This shows the level of direct production that is necessary to keep the consumed service level $z$ for a given set of population $n$ and local characteristics $\boldsymbol{a}$. To see the effects of population on the public expenditures, we substitute (4) into (2) to obtain:

$$
c=c(\gamma(z, n, \boldsymbol{a}), \boldsymbol{w}) \text {. }
$$

Returns to scale are defined in terms of changes in average cost when the level of an "output" varies. As taxonomized in Duncombe and Yinger (1993), there are three dimensions for returns to scale in local public production: (i) technical returns to scale over $g$, (ii) returns to population scale over $n$, and (iii) returns to quality scale over $z$. The "U-shaped" per capita expenditure is associated with the second concept and characterized by the partial derivative:

$$
\frac{\partial}{\partial n} \cdot\left[\frac{c(\gamma(z, n, \boldsymbol{a}), \boldsymbol{w})}{n}\right]
$$

In fact, with a proper rearrangement, we can show that 


$$
\frac{\partial(c / n)}{\partial n}=\frac{c / n}{n} \cdot\left(\frac{\eta_{n}^{g}}{\eta_{c}^{g}}-1\right)
$$

where

$$
\begin{aligned}
& \eta_{n}^{g} \equiv \frac{\partial \gamma}{\partial n} \cdot \frac{n}{g} \text { and } \\
& \eta_{c}^{g} \equiv \frac{\partial g}{\partial c} \cdot \frac{c}{g} .
\end{aligned}
$$

This shows that the direction of population-induced changes in per capita cost is related to the relative magnitude between the two elasticities, $\eta_{n}{ }^{g}$ and $\eta_{c}{ }^{g}$.

The first elasticity (7) is a congestion elasticity characterized by the consumption technology (3)-(4). It measures the elasticity of $g$ with respect to $n$, keeping $z$ and $\boldsymbol{a}$ constant, and shows how much direct product needs to be increased in order to keep the service level as before, when population increases. On the other hand, the very definition that congestion refers to a negative impact of a population increase on the level of public service may imply

$$
\eta_{n}^{z} \equiv-\frac{\partial z}{\partial n} \cdot \frac{n}{g}
$$

which is the elasticity of $z$ with respect to $n$ (in absolute value), keeping $g$ and $\boldsymbol{a}$ constant. However, (7) outperforms (9) in that the former is metric-free while the latter is not (Reiter and Weichenrieder 1999). Note that as only ordering is required when $z$ is evaluated by citizen-consumers, any variable that is a positive monotone transformation of $z$ can validly replace $z$. However, (9) is invariant only to multiplicative changes of $z$. As such, when we employ (9) rather than (7), the value of the elasticity will arbitrarily differ depending on the monotonic transformation.

The second elasticity (8) is technical elasticity of scale, which is associated with the production technology (1). Increasing technical returns to scale (or technical economies of scale) imply that $\partial(c / g) / \partial g<0$. A natural expression for its elasticity will then be given as $\eta_{g} \equiv-[\partial(c / g) / \partial g] \cdot[g /(c / g)]$, which can be related to (8) as:

$$
\eta_{g}=1-\frac{1}{\eta_{c}^{g}}
$$

This shows that the values of $\eta_{c}^{g}$ and $\eta_{g}$ are monotone, allowing us to regard the former 
as a valid measure for technical economies of scale.

Eq. (6), in fact, is a different restatement of what is derived in Duncombe and Yinger (1993). ${ }^{1}$ Our articulation, however, may be more instructive. First, we may easily relate changes in per capita expenditures to the relative magnitudes between $\eta_{n}{ }^{g}$ and $\eta_{c}{ }^{g}$. Specifically, per capita cost is decreasing (or returns to population scale are increasing) when technical elasticity of scale is greater than congestion elasticity $\left(\eta_{c}^{g}>\right.$ $\eta_{n}{ }^{g}$ ). Furthermore, per capita cost is increasing (or returns to population scale are decreasing), when technical elasticity of scale is less than congestion elasticity $\left(\eta_{c}^{g}<\right.$ $\eta_{n}{ }^{g}$ ). While a typical explanation in the literature relates U-shaped per-capita local expenditures to some returns to scale and congestion, eq. (6) indeed formalizes the fact that the U-shaped curve hinges on the balance between the two measures, $\eta_{n}{ }^{g}$ and $\eta_{c}{ }^{g}$.

Second, our expression helps construct an indicator that quantifies a deviation of a local population from its MES for local public expenditures. By multiplying both sides of eq. (6) by $n /(c / n)$, we obtain an elasticity expression for the population returns to scale:

$$
\frac{\partial(c / n)}{\partial n} \cdot \frac{n}{c / n}=\frac{\eta_{n}^{g}}{\eta_{c}^{g}}-1
$$

We may then define an efficiency indicator for a local population as:

$$
S(n) \equiv \frac{\eta_{n}^{g}}{\eta_{c}^{g}}
$$

We then see from eq. (6) that $n^{*}$, such that $S\left(n^{*}\right)=1$ (i.e., $\eta_{n}{ }^{g}=\eta_{c}{ }^{g}$ ), is the MES, provided that (i) both $\eta_{n}{ }^{g}$ and $\eta_{c}^{g}$ are not constant, (ii) $\eta_{n}{ }^{g}$ is non-decreasing in $n$, and (iii) $\eta_{c}{ }^{g}$ is non-increasing in $n$. We also see from eq. (6) that a local population is too large (small) if $S(n)>(<) 1$. Thus, we may treat $S(n)$ as an indicator that quantifies the deviation of a local population size from its MES.

Notice that the MESs differ across jurisdictions in so far as the local factors that influence the two elasticities also differ. Those factors generally consist of the variables that appear in eq. (5). However, they do not necessarily include all of the variables,

\footnotetext{
${ }^{1}$ Duncombe and Yinger (1993) use an elasticity expression that is analogous to eq. (10). Their expression is $[\partial(c / n) / \partial n] \cdot n /(c / n)=\eta_{c}{ }^{g} \cdot \eta_{n}{ }^{g}-1$, where the elasticity for technical returns to scale is defined differently as $\eta_{c}^{g} \equiv \partial c / \partial g \cdot g / c$, a reciprocal of our measure.
} 
which depend on the specifications of the production technology (1).

\section{Empirical Implementation}

In this section, on the basis of the foregoing analysis, we estimate the urban public production structure in Japan. With the estimates obtained, we calculate the efficient indicators for the cities whose data are used for the estimation and rank them according to the resultant values of the calculated scores.

\subsection{Specifications}

To implement the foregoing analysis, we need to estimate the technical elasticity of scale and congestion elasticity of population. To do so, we have to specify the cost function (2) and the congestion function (3). Let us start with the congestion function. A popular specification for the congestion function is $z=n^{-\alpha} g$ (Borcherding and Deacon 1972, Bergstrom and Goodman 1973). This, in effect, assumes that congestion decreases at the margin, and its elasticities are constant at $\alpha$. However, congestion may or may not accelerate with the intensity of use, and its elasticities should not be constant. In addition, as discussed in the previous section, factors other than population may influence congestion. We thus specify our congestion function as:

$$
z=g \cdot n^{-\left(\lambda_{0}+\lambda_{n} \ln n+\sum_{j} \lambda_{j} a_{j}\right)} \cdot \exp \left\{-\sum_{j} \delta_{j} a_{j}\right\}
$$

where $a_{j}$ represents environment factors (i.e., is an element of vector $\boldsymbol{a}$ ), and $\lambda_{j}$ and $\delta_{j}$ are parameters to be estimated.

The following points must be noted. First, our specification of the congestion function is a proportional metric: $z(g, n, \boldsymbol{a})=g \cdot \xi(n, \boldsymbol{a})$. Eq. (3) yields $\eta_{n}{ }^{g}=\eta_{n}{ }^{z} / \eta_{g}{ }^{z}$, where $\eta_{g}{ }^{z} \equiv(\partial z / \partial g) \cdot(g / z)$. Since $\eta_{g}{ }^{z}=1$ if $z(g, n, \boldsymbol{a})=g \cdot \xi(n, \boldsymbol{a})$, it is immediate that the two congestion elasticities (7) and (9) coincide (cf., Reiter and Weichenrieder 1999). Second, we can test whether congestion elasticity is increasing or decreasing. Given specification (13), the congestion elasticity is given as $\eta_{n}{ }^{g}=\lambda_{0}+2 \lambda_{n} \cdot \ln n+\sum \lambda_{j} \cdot a_{j}$, which enables us to test whether congestion is increasing or decreasing by examining the sign of $\lambda_{n}$ (e.g., Hayes and Slottje 1987). Second, we allow the environmental factors to affect the elasticity through $\sum \lambda_{j} \cdot a_{j}$ (e.g., Hayes 1986, Duncombe and Yinger 
1993). Note that this also allows for the possibility of a "camaraderie" effect (Edward 1990), since if some of the $\lambda_{0} \mathrm{~s}$ and $\lambda_{j} \mathrm{~s}$ are negative, sharing congestible goods may actually increase benefits to each user for a given combination of values of the environmental factors. ${ }^{2}$ Finally, we include $\exp \left\{-\sum \delta_{j} \cdot a_{j}\right\}$ in (12) so that the environmental factors multiplicatively influence the level of public services.

Following the convention of the literature, we aggregate factor inputs into labor and capital, which is reflected as two factor prices, $w$ for labor and $r$ for capital, in the cost function. As a specification for the cost function, we first assume the translog form:

$$
\begin{aligned}
\ln c & =\beta_{0}+\beta_{g} \ln g+\beta_{w} \ln w+\beta_{r} \ln r+\beta_{g g} \ln g \cdot \ln g+\beta_{g w} \ln g \cdot \ln w+\beta_{g r} \ln g \cdot \ln r \\
& +\beta_{w w} \ln w \cdot \ln w+\beta_{w r} \ln w \cdot \ln r+\beta_{r r} \ln r \cdot \ln r
\end{aligned}
$$

where the $\beta$ s are parameters to be estimated. Note that the price of the capital $r$ could be regarded as uniform across regions within a country in a given period (e.g., Kitchen 1976, Stevens 1978). As such, we can forego obtaining the data for $r$ since our data are cross-sectional. This means that some of the coefficients are not identifiable, and the feasible specification is reduced to

$$
\ln c=A_{0}+A_{w} \ln w+\beta_{w w} \ln w \cdot \ln w+\left(A_{g}+\beta_{g w} \ln w\right) \cdot \ln g+\beta_{g g} \ln g \cdot \ln g
$$

where coefficients $A_{0}, A_{g}$, and $A_{w}$ correspond respectively to $\beta_{0}+\beta_{r} \cdot \ln r+\beta_{r r} \cdot \ln r \cdot \ln r, \beta_{g}$ $+\beta_{g r} \cdot \ln r$, and $\beta_{w}+\beta_{w r} \cdot \ln r$ in the original specification.

We next obtain the log for (13) and rearrange the resultant terms to obtain:

$$
\ln g=\ln z+\left(\lambda_{0}+\lambda_{n} \ln n+\sum_{j} \lambda_{j} a_{j}\right) \cdot \ln n+\sum_{j} \delta_{j} a_{j} .
$$

By substituting this into (14), indexing its variables with subscript $i$, and adding error term $u_{i}$, we finally obtain the following statistical specification for the cost function:

$$
\begin{aligned}
\ln c_{i} & =A_{0}+A_{w} \ln w_{i}+\beta_{w w} \ln w_{i} \cdot \ln w_{i} \\
& +\left(A_{g}+\beta_{g w} \ln w_{i}\right) \cdot\left[\ln z_{i}+\left(\lambda_{0}+\lambda_{n} \ln n_{i}+\sum_{j} \lambda_{j} a_{j i}\right) \cdot \ln n_{i}+\sum_{j} \delta_{j} a_{j i}\right] \\
& +\beta_{g g} \cdot\left[\ln z_{i}+\left(\lambda_{0}+\lambda_{n} \ln n_{i}+\sum_{j} \lambda_{j} a_{j i}\right) \cdot \ln n_{i}+\sum_{j} \delta_{j} a_{j i}\right]^{2}+u_{i} .
\end{aligned}
$$

We can test the forms of production technology by imposing coefficient restrictions. Specifically, we test the Cobb-Douglas form against the translog specification with restriction $\beta_{w w}=\beta_{g w}=\beta_{g g}=0$. If the restrictions are valid, the specification will be:

\footnotetext{
2 While Edwards (1990) considers the question of increasing congestion and that of "camaraderie" effects by setting up separate specifications, our single specification allows for both possibilities.
} 


$$
\begin{aligned}
& \ln c_{i}=B_{0}+B_{w} \ln w_{i} \\
& \quad+\beta_{g} \cdot\left[\ln z_{i}+\left(\lambda_{0}+\lambda_{n} \ln n_{i}+\sum_{j} \lambda_{j} a_{j i}\right) \cdot \ln n_{i}+\sum_{j} \delta_{j} a_{j i}\right]+u_{i}
\end{aligned}
$$

where $B_{0}=\beta_{0}+\beta_{r} \cdot \ln r$.

\subsection{Data}

We need data for total cost $c_{i}$, population $n_{i}$, wages for local public officials $w_{i}$, public service consumed $z_{i}$, and a set of environmental factors $\boldsymbol{a}$. The data we utilize are obtained from a cross section of Japanese cities in 1995. Although there are more than 600 cities in Japan in that year, the availability of the variables used for the estimation reduces the sample size down to 572. The data descriptions and their sources are listed in Table 1. The details of each variable are explained as follows.

Total cost and population: We examine the cost for overall public services provided by city governments. The total cost $c_{i}$ is naturally represented by the total expenditures of a city government, listed in the Ministry of Internal Affairs and Communication (MIC) (1997). Our data for population $n_{i}$ are drawn from the Statistics Bureau, Management and Coordination Agency (1997).

Wages: The price of public labor $w_{i}$ is obtained as average wages, that is, the compensation of public employees divided by the number of public employees (MIC 1997). This implicitly assumes that the average working hours are identical across the cities under examination, which may be justified given the fact that labor conditions for local public employees seem to be similar across municipalities.

Public service outputs: Obtaining the data for the consumed level of total public service $z_{i}$ is not straightforward. We use the "total score of public services" provided by Nihon Keizai Shinbunsya (1998). This score quantifies the overall quality level of Japanese local government outputs, by considering the total of 24 public service categories, which include fees for charged public services, per capita capacities of welfare and education facilities, and per capita levels of infrastructures like roads, parks, water supply, and sewage facilities. The data for each category are transformed to standardized values. If the standardized value is less than 20 , only 1 point is given; $20-$ 40, 2 points; 40-60, 3 points; $60-80,4$ points; and 80 and over, 5 points. The final score for a jurisdiction is the total of these points scored in the 24 categories. While this score may not be a good measure for the actual quality of the public services, we believe it 
should give us a reasonable evaluation of local public services. In addition, it is the only available data for public service quality.

Environmental factors: The environmental factors fall into four groups: physical factors, urban factors, demographic factors, and economic factors. We try to capture the physical environment by considering the total land area in $\log$ form $\left(a_{1}\right)$, as well as the following shares of sub-areas: "distant-island" area $\left(a_{2}\right)$, "heavy-snowfall" area $\left(a_{3}\right)$, forested area $\left(a_{4}\right)$, and farm lands $\left(a_{5}\right)$. The "distant-island" and "heavy-snowfall" areas are subdivisions in a jurisdiction designated as such by national laws, which should be a convenient set of indicators for harsh natural environments. Note that the number of the subdivisions is used to calculate shares $a_{2}$ and $a_{3}$. For example, if a city has five subdivisions and one of them is designated as "distant-island," then the share is $1 / 5$. For $a_{4}$ and $a_{5}$, on the other hand, we simply use areas to compute shares, that is, percentages of forested area and farm land in the total area of a city. As urban factors, we consider three elements. They are the proportion of population in Densely Inhabited Districts (DID) population $\left(a_{6}\right)$, the share of the areas that DIDs cover $\left(a_{7}\right)$, and the ratio of daytime population to nighttime population $\left(a_{8}\right)$. For demographic factors, we only consider percentages of the young, that is, those aged less than 16 years $\left(a_{9}\right)$, and the elderly, that is, those aged above 64 years $\left(a_{10}\right)$. Finally, economic factors are controlled by the log of per capita income $\left(a_{11}\right)$, and the proportions of workers engaged in the manufacturing $\left(a_{12}\right)$ and service sectors $\left(a_{13}\right)$.

Additional dummies: Although not explicitly written in (14a) or (14b), we add three dummy variables that affect the intercept of the regression function $\left(A_{0}\right.$ or $\left.B_{0}\right)$. Two dummies are intended to capture the different administrative roles assumed by two special types of Japanese cities. One, $\left(D_{1}\right)$, is for a group of 12 designated cities that have a longer list of expenditure authorities than ordinary cities do. Another, $\left(D_{2}\right)$, is for 23 special wards $(k u)$ in Tokyo, which delegates some of their expenditure responsibilities to the Tokyo metropolitan government. ${ }^{3}$ The last is the dummy for the Great Kobe Earthquake in January $1995\left(D_{3}\right)$. While our fiscal data are for the 1995 fiscal year (April 1995 to March 1996) and do not cover the time of the earthquake, it is

\footnotetext{
${ }^{3}$ For example, police and refuse collection are administrated by municipalities (i.e., cities, towns, and villages) in the standard case. However, in the case of the special wards, they are taken care of by the Tokyo metropolitan government and the upper tier of the special wards (note that refuse collection has now become a responsibility of the special districts, starting in 2000).
} 
plausible that the damages were so serious that local public expenditures in the inflicted areas in the following fiscal year increased owing to the special needs caused by the disaster. We thus include a dummy variable that indicates such inflicted areas.

Table 1. Data description

\begin{tabular}{c|l|c}
\hline \hline Variables & \multicolumn{1}{|c}{ Description } & Source \\
\hline$c$ & Total public expenditures & $\mathrm{A}$ \\
$n$ & Population & $\mathrm{B}$ \\
$w$ & Average wages for public employees & $\mathrm{A}$ \\
$z$ & Total score of public services & $\mathrm{D}$ \\
$a_{1}$ & Log of total area of a city & $\mathrm{B}$ \\
$a_{2}$ & Ratio of "distant-island area" & $\mathrm{E}$ \\
$a_{3}$ & Ratio of "heavy-snow area" & $\mathrm{E}$ \\
$a_{4}$ & Ratio of forested area & $\mathrm{E}$ \\
$a_{5}$ & Ratio of farm land & $\mathrm{E}$ \\
$a_{6}$ & Ratio of DID population & $\mathrm{B}$ \\
$a_{7}$ & Ratio of DID & $\mathrm{B}$ \\
$a_{8}$ & Ratio of daytime population & $\mathrm{B}$ \\
$a_{9}$ & Ratio of 15 years or younger & $\mathrm{B}$ \\
$a_{10}$ & Ratio of 65 years or older & $\mathrm{B}$ \\
$a_{11}$ & Log of per capita income & $\mathrm{F}$ \\
$a_{12}$ & Ratio of manufacturing labor & $\mathrm{B}$ \\
$a_{13}$ & Ratio of service labor & $\mathrm{B}$ \\
$D_{1}$ & Designated cities (dummy) & - \\
$D_{2}$ & Special wards (dummy) & - \\
$D_{3}$ & Great Kobe Earthquake (dummy) & - \\
\hline
\end{tabular}

Sources: a: Ministry of Internal Affairs and Communication; b Statistic Bureau, Management and Coordination Agency; c: National Land Agency; d: Nihon Keizai Shinbunsya; e: Ministry of Agriculture, Forestry and Fishery; f: Ministry of Finance. Note that DID stands for "Densely Inhabited Districts."

\subsection{Specification tests}

Since we use cross section data from a variety of cities, we may legitimately assume that the error term $u_{i}$ follow some pattern of heteroskedasticity. However, as there are several variables to consider in our nonlinearly specified regression functions, determining a specific pattern of the skedastic function is a difficult task. We therefore assume that the pattern of the heteroskedasticity is unknown, and employ econometric methods that allow for unknown patterns of heteroskedasticity in the ensuing analysis. Before presenting the final estimates, we perform specification tests to choose between 
(14a) and (14b) to determine the type of production technology (i.e., translog or Cobb-Douglas functions). The null hypothesis is that the technology is a Cobb-Douglas function $\left(\beta_{w w}=\beta_{g w}=\beta_{g g}=0\right)$. If the null hypothesis is not rejected, we opt for $(14 \mathrm{~b})$. Otherwise, (14a) is chosen. Our test is based upon the Lagrange multiplier (LM) principle. To construct relevant LM test statistics, we utilize the Gauss-Newton regression (GNR) that allows for heteroskedasticity, that is, the heteroskedasticityrobust Gauss-Newton regression (HRGNR). ${ }^{4}$ The HRGNR shows that the CobbDouglas technology is not rejected at the standard significance levels with a $P$ value of .115 . We thus base our discussion on (14b), whose estimates are obtained by the method of non-linear least squares, as discussed in the following section. ${ }^{5}$

\section{Results}

The estimation results for (14b) are listed in Table 2, where the coefficients for $D_{1}, D_{2}$, and $D_{3}$ are indicated as $b_{1}, b_{2}$, and $b_{3}$, respectively. Note that $P$ values are based upon the heteroskedasticity consistent covariance matrix estimator (HCCME). The estimates for the production technology, namely $B_{0}, \beta_{w}$, and $\beta_{g}$, have the expected signs and are all statistically significant. Also, the latter two estimates are within the range suggested by the theory. Note that since we have opted for the Cobb-Douglas technology, the values for technical elasticity of scale $\eta_{c}{ }^{g}$ are constant and equal to the reciprocal of $\eta_{g}$. With $\beta_{g}=.328$, the value of the elasticity is $\eta_{c}^{g}=1 / \beta_{g}=3.049$, which implies that $\partial(c / g) / \partial g<0$ by eq. (10). That is, the technical returns to scale are increasing and technical economies of scale exist in the Japanese local public production.

The estimate for $\lambda_{n}$ is positive and statistically significant at the significance levels larger than .011. Given the congestion elasticity $\eta_{n}{ }^{g}=\lambda_{0}+2 \lambda_{n} \cdot \ln n_{i}+\sum \lambda_{j} \cdot a_{j i}$, this positive value implies that congestion accelerates as population increases. Note that since the values for the $\lambda_{0}$ s and some $\lambda_{j}$ s are negative, the value of $\eta_{n}{ }^{g}$ can be negative if $2 \lambda_{n} \cdot \ln n_{i}<-\lambda_{0}-\sum \lambda_{j} \cdot a_{j i}$, allowing for the possibility of the camaraderie effect. We

\footnotetext{
${ }^{4}$ See Davidson and MacKinnon (1993, pp. 399-402) for a textbook explication, and the references thereof for further details.

${ }^{5}$ Note that we assume the output variable $z_{i}$ is independent of total $\cos t c_{i}$ and therefore of error term $u_{i}$, which is a common assumption in the literature. The basis for this assumption is a public choice process where some public decision makers (e.g., voters) decide the desired level of output, which is exogenous to public officials who minimize costs subject to their technical constraint.
} 
calculate the congestion elasticities for the 572 cities that constitute the observations in our sample. Table 3 reports the summary statistics of the $572 \eta_{n}{ }^{g}$ s which, with a minimum of 1.269 , show no evidence of the camaraderie effect.

Table 2. Estimation results

\begin{tabular}{|c|c|c|c|c|c|}
\hline Coef. & Estimates & P value & Coef. & Estimates & $P$ value \\
\hline$B_{0}$ & $21.9417^{* * *}$ & .0000 & & & \\
\hline$\beta_{w}$ & $.1790^{* *}$ & .0348 & & & \\
\hline$\beta_{g}$ & $.3280^{* * *}$ & .0023 & & & \\
\hline$b_{1}$ & $.2723^{* * *}$ & .0003 & & & \\
\hline$b_{2}$ & .0572 & .3714 & & & \\
\hline$b_{3}$ & $.2807^{* * *}$ & .0006 & & & \\
\hline$\lambda_{n}$ & $.3746^{* *}$ & .0111 & & & \\
\hline$\lambda_{0}$ & $-8.2535^{* *}$ & .0118 & & & \\
\hline$\lambda_{1}$ & $-.2424^{* *}$ & .0308 & $\delta_{1}$ & $3.0124^{* *}$ & .0234 \\
\hline$\lambda_{2}$ & -.1665 & .4799 & $\delta_{2}$ & 2.0120 & .4136 \\
\hline$\lambda_{3}$ & .0137 & .8410 & $\delta_{3}$ & .0131 & .9865 \\
\hline$\lambda_{4}$ & $.7016^{*}$ & .0780 & $\delta_{4}$ & $-8.2502^{*}$ & .0750 \\
\hline$\lambda_{5}$ & .7614 & .2066 & $\delta_{5}$ & -8.9980 & .1936 \\
\hline$\lambda_{6}$ & -.2465 & .3968 & $\delta_{6}$ & 3.0192 & .3619 \\
\hline$\lambda_{7}$ & -.1744 & .5333 & $\delta_{7}$ & 2.5475 & .4304 \\
\hline$\lambda_{8}$ & $.2539^{* * *}$ & .0063 & $\delta_{8}$ & $-2.5510^{* * *}$ & .0066 \\
\hline$\lambda_{9}$ & $5.3103^{*}$ & .0903 & $\delta_{9}$ & -56.6047 & .1059 \\
\hline$\lambda_{10}$ & $4.5159^{* *}$ & .0327 & $\delta_{10}$ & $-45.1757^{* *}$ & .0464 \\
\hline$\lambda_{11}$ & .2025 & .3331 & $\delta_{11}$ & -2.4509 & .3143 \\
\hline$\lambda_{12}$ & 1.7067 & .1626 & $\delta_{12}$ & -18.1052 & .1657 \\
\hline$\lambda_{13}$ & 1.2898 & .2697 & $\delta_{13}$ & -13.2239 & .2884 \\
\hline \multicolumn{2}{|c|}{ Adjusted $R^{2}$} & \multicolumn{4}{|c|}{.974} \\
\hline \multicolumn{2}{|c|}{ Number of observations } & \multicolumn{4}{|c|}{572} \\
\hline \multicolumn{2}{|c|}{ Degree of freedom } & \multicolumn{2}{|c|}{538} & & \\
\hline
\end{tabular}

Table 3. Summary statistics of congestion elasticities for $\mathbf{5 7 2}$ cities

\begin{tabular}{ccccc}
\hline \hline Congestion Elasticity & Mean & Std. Error. & Minimum & Maximum \\
\hline$\eta_{n}^{g}$ & 2.6069 & .5613 & 1.2692 & 8.5844 \\
\hline
\end{tabular}


Several, but not all, of the coefficients on the environment factors $\left(\lambda_{j} \mathrm{~s}\right.$ and $\left.\delta_{j} \mathrm{~s}\right)$ are statistically significant, implying that the degree of congestion depends on factors other than population. It is also interesting to note that the environmental factors that have significant effects through $\delta_{j}$ also do so through $\lambda_{j}$. These factors are the city's total area, share of forested area, daytime population ratio, and elder population ratio. Note that the effect of a marginal change in one of the environmental factors is not easily read from the coefficient estimates, since it also depends on the values of $\beta_{w}$ and $\ln n_{i}$ :

$$
\frac{\partial \ln c_{i}}{\partial a_{j i}}=\beta_{w} \cdot\left(\lambda_{j} \ln n_{i}+\delta_{j}\right) .
$$

We calculate $\partial \ln c_{i} / \partial a_{j i}$ for the 572 cities for each environmental factor. Table 4 provides the summary statistics, which clearly show that cities with different populations implies different and sometimes opposite effects of the environmental factors.

Table 4. Marginal effects of the environmental factors

\begin{tabular}{c|c|c|c|c}
\hline \hline Effects & Mean & Std. Error. & Minimum & Maximum \\
\hline$\partial \ln c_{i} / \partial a_{1 i}$ & .0801 & .0735 & -.2057 & .2368 \\
$\partial \ln c_{i} / \partial a_{2 i}$ & .0362 & .0505 & -.1601 & .1438 \\
$\partial \ln c_{i} / \partial a_{3 i}$ & .0556 & .0042 & .0468 & .0718 \\
$\partial \ln c_{i} / \partial a_{4 i}$ & -.0784 & .2127 & -.5319 & .7485 \\
$\partial \ln c_{i} / \partial a_{5 i}$ & -.0997 & .2308 & -.5919 & .7977 \\
$\partial \ln c_{i} / \partial a_{6 i}$ & .0671 & .0747 & -.2234 & .2264 \\
$\partial \ln c_{i} / \partial a_{7 i}$ & .1823 & .0529 & -.0233 & .2950 \\
$\partial \ln c_{i} / \partial a_{8 i}$ & .1141 & .0770 & -.0500 & .4133 \\
$\partial \ln c_{i} / \partial a_{9 i}$ & 1.3221 & 1.6099 & -2.1105 & 7.5806 \\
$\partial \ln c_{i} / \partial a_{10 i}$ & 2.0958 & 1.3690 & -0.8233 & 7.4181 \\
$\partial \ln c_{i} / \partial a_{11 i}$ & -.0455 & .0614 & -.1764 & .1931 \\
$\partial \ln c_{i} / \partial a_{12 i}$ & .4534 & .5174 & -.6498 & 2.4648 \\
$\partial \ln c_{i} / \partial a_{13 i}$ & .4933 & .3910 & -.3405 & 2.0134 \\
\hline
\end{tabular}

Lastly, the coefficient estimates for the dummies for the designated cities $\left(b_{1}\right)$ and the Kobe Earthquake $\left(b_{3}\right)$ have the expected signs, as they both tend to increase local public expenditures. The positive sign of the coefficient for the special wards in Tokyo $\left(b_{2}\right)$ are unexpected, since they have less administrative responsibilities than the ordinary cities. However, the estimate is not significant at the standard levels of significance.

Table 5 shows the summary statistics of the scale index $S(n) \equiv \eta_{n}{ }^{g} / \eta_{c}^{g}$ calculated 
for the 572 cities. Since the value for $\eta_{c}{ }^{g}$ is fixed at $1 / \beta_{w}=3.049$, variations in $S(n)$ across the cities are due to those in $\eta_{n}{ }^{g}$, which in turn depend on local population as well as other environmental factors $\left(\eta_{n}{ }^{g}=\lambda_{0}+2 \lambda_{n} \cdot \ln n_{i}+\sum \lambda_{j} \cdot a_{j i}\right)$. The rank correlation coefficient between $S(n)$ and $n$ is .921 , which appears to be rather high despite the inclusion of the environmental factors in the formula for $\eta_{n}{ }^{g}$. This may be because some of the environmental factors like the DID ratio and per capita income are highly correlated with population. Figure 1 shows the cumulative distribution of the scale index. Since population $n^{*}$, such that $S(n)=1$ is MES, about $81 \%$ of the 572 cities is regarded as underpopulated whereas only $19 \%$ are regarded as overpopulated. To give a preview of the results, Table 6 lists the top 20 cities ranked according to the scale index along with their population ranks. In addition, Table 7 shows the cities in the vicinity of the optimal size with $S(n)$ such that $.990<S(n)<1.001$.

Table 5. Summary statistics of scale index values for 572 cities

\begin{tabular}{c|c|c|c|c}
\hline \hline & Mean & Std. Error. & Minimum & Maximum \\
\hline$S(n)$ & .8551 & .1841 & .4163 & 2.8158 \\
$\eta_{n}$ & -.1449 & .1841 & -.5837 & 1.8158 \\
\hline
\end{tabular}

Figure 1. Distribution of cities according to the scale index

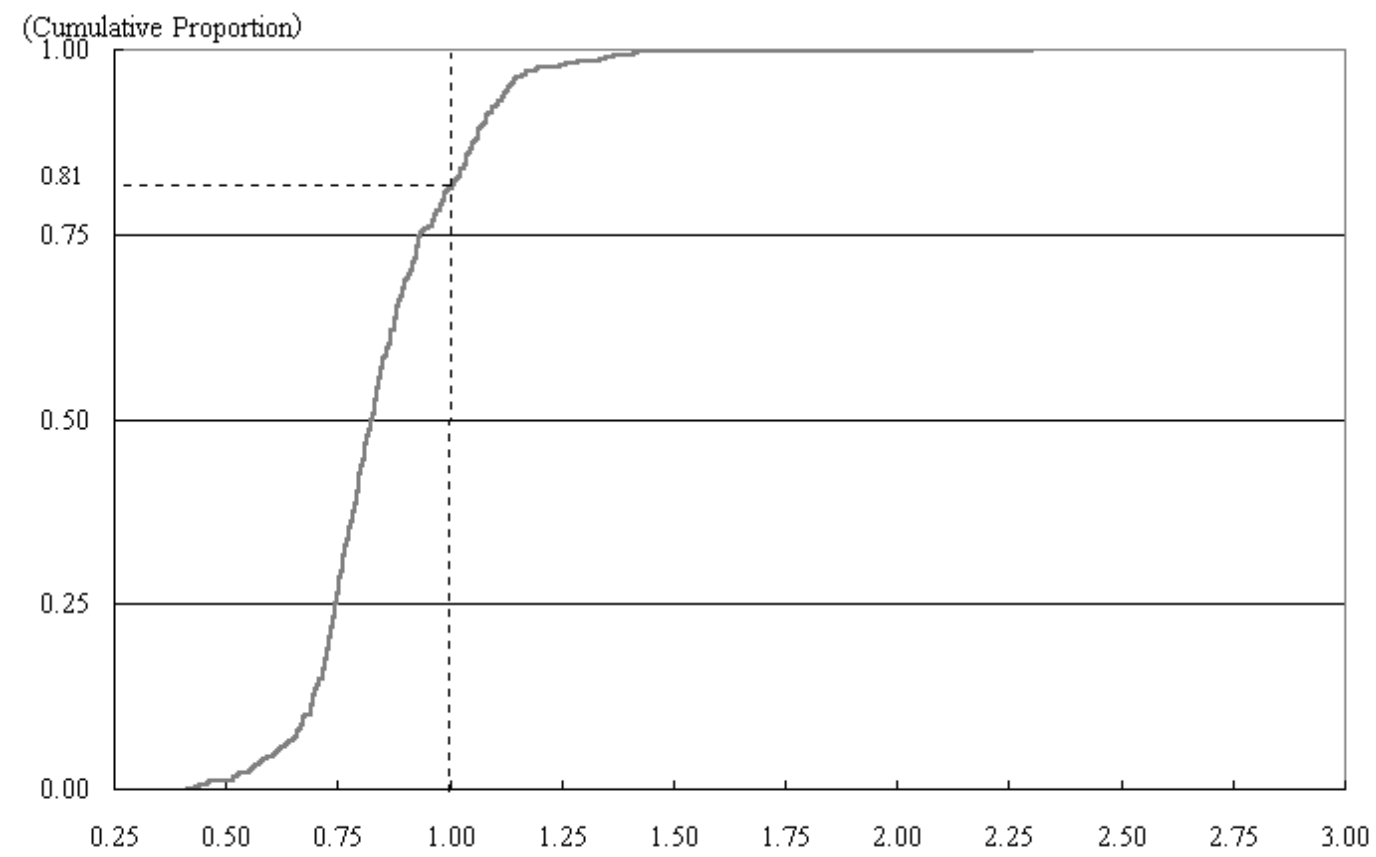


Table 6. Top 20 overcongested cities

\begin{tabular}{c|l|r|r|r}
\hline Rank & \multicolumn{1}{|c|}{ Name } & $S(n)$ & Pop. Rank & Population \\
\hline 1 & Chiyoda (Tokyo) & 2.8158 & 504 & 34.8 \\
2 & Chuo (Tokyo) & 1.5700 & 329 & 63.9 \\
3 & Yokohama & 1.4266 & 1 & $3,307.1$ \\
4 & Osaka & 1.4180 & 2 & $2,602.4$ \\
5 & Kyoto & 1.3715 & 5 & $1,463.8$ \\
6 & Nagoya & 1.3597 & 6 & $2,152.2$ \\
7 & Kobe & 1.3337 & 4 & $1,423.8$ \\
8 & Sapporo & 1.3080 & 9 & $1,757.0$ \\
9 & Hiroshima & 1.2906 & 7 & $1,108.9$ \\
10 & Fukuoka & 1.2844 & 154 & $1,284.8$ \\
11 & Minato (Tokyo) & 1.2787 & 10 & 144.9 \\
12 & Kitakyusyu & 1.2453 & 11 & $1,019.6$ \\
13 & Sendai & 1.2415 & 15 & 971.3 \\
14 & Kawasaki & 1.2071 & 19 & $1,202.8$ \\
15 & Kumamoto & 1.1919 & 22 & 650.3 \\
16 & Okayama & 1.1781 & 23 & 615.8 \\
17 & Hamamatsu & 1.1733 & 39 & 561.6 \\
18 & Kagoshima & 1.1700 & 12 & 546.3 \\
19 & Nagasaki & 1.1682 & 438.6 \\
20 & Chiba & & 856.9 \\
\hline
\end{tabular}

Note: Populations are in thousands. Chiyoda, Chuo, and Minato are special wards in the Tokyo Metropolis.

Table 7. Minimally efficient cities

\begin{tabular}{c|l|r|r|r}
\hline \hline Rank & \multicolumn{1}{|c|}{ Name } & $S(n)$ & Pop. Rank & Population \\
\hline 104 & Nagaoka & 1.0093 & 118 & 190.5 \\
105 & Suita & 1.0088 & 59 & 342.8 \\
106 & Takarazuka & 1.0086 & 110 & 202.5 \\
107 & Hitachi & 1.0084 & 115 & 119.2 \\
108 & Odawara & 1.0043 & 113 & 200.1 \\
109 & Kashiwa & 1.0012 & 70 & 317.8 \\
110 & Nakano (Tokyo) & .9942 & 72 & 306.6 \\
111 & Meguro (Tokyo) & .9911 & 96 & 243.1 \\
112 & Kishiwada & .9910 & 117 & 194.8 \\
113 & Ishihara & .9900 & 85 & 277.1 \\
\hline
\end{tabular}

Note: The ranks in the first column are in terms of $S(n)$. Populations are in thousands. Nakano and Meguro are special wards in the Tokyo Metropolis. 


\section{Concluding Remarks}

In this paper, we analytically delineated the factors that account for the "U-shaped" per capita local public expenditures. The important concepts examined are (i) technical elasticity of scale, which characterizes technology for the direct outputs produced by a government, and (ii) congestion elasticity, which characterizes consumption technology for the public service level consumed by citizens. By articulating that population-induced changes in per capita cost are related to the relative magnitude between the two elasticities, we constructed an indicator that quantifies the distance of a local population from its MES for local public expenditures. We then estimated the urban public production structure in Japan and applied the analysis to the Japanese case. With the estimates obtained, we ranked the Japanese cities according to the calculated values of the indicator.

\section{References}

Borcherding, T.E., Deacon, R.T., 1972. The demand for the services of non-federal government. American Economic Review 62, 891-901.

Bergstrom, T.C., Goodman, R.P., 1973. Private demands for public goods. American Economic Review 63, 280-296.

Bradford, D.F., Malt, R.A., Oates, W.E., 1969. The rising cost of local public services: Some evidence and reflections. National Tax Journal 22, 185-202.

Brueckner, J.K, 1981. Congested public goods: The case of fire protection. Journal of Public Economics 15, 45-58.

Davidson, R., MacKinnon, J.G., 1993. Estimation and Inference in Econometrics, Oxford University Press, New York.

Duncombe, W., Yinger, J., 1993. An analysis of returns to scale in public production, with an application to fire protection. Journal of Public Economics 52, 49-72.

Edwards, J.H.Y., 1990. Congestion function specification and the "publicness" of local public goods. Journal of Urban Economics 27, 80-96.

Hayes, K., 1986. Local public good demand and demographic effects. Applied Economics 18, 1039-1045.

Hayes, K.J., Slottje, D.J., 1987. Measure of publicness based on demographic scaling. Review of Economics and Statistics 69, 713-718.

Hirsch, W.Z., 1959. Expenditure implications of metropolitan growth and consolidation. Review of Economics and Statistics 41, 232-241.

Hirsch, W.Z., 1965. Cost functions of an urban government service: Refuse collection. Review of Economics and Statistics 47, 87-93.

Kitchen, H., 1976. A statistical estimation of an operating cost function for municipal refuse collection. Public Finance Quarterly 4, 56-76. 
Ministry of Internal Affairs and Communication, 1997. Shichoson Kessan Jokyo Shirabe 1995 (Local Public Expenditures of Municipalities 1997).

Nihon Keizai Shinbunsya, 1998. 610 shi no gyosei sahbisu suijun ichi-ran (Table of public service levels of 610 cities). Nikkei Chi-iki Joho, 0(303).

Reiter, M., Weichenrieder, A.J., 1999. Public goods, club goods, and the measurement of crowding. Journal of Urban Economics 46, 69-79.

Statistics Bureau, Management and Coordination Agency, 1997. Kokusei Chosa 1995 (National Census 1995).

Stevens, B.J., 1978. Scale, market structure, and the cost of refuse collection. Review of Economics and Statistics 60, 438-448. 